\title{
EXPERIMENTAL STUDY OF THE CHAOTIC JERK CIRCUIT APPLICATION FOR CHAOS SHIFT KEYING
}

\author{
F. Capligins*, A. Litvinenko**, A. Aboltins, \\ E. Austrums, A. Rusins, D. Pikulins \\ Faculty of Electronics and Telecommunications, \\ Institute of Radioelectronics, Riga Technical University, \\ 12 Azenes Str., Riga, LV-1048, LATVIA \\ *e-mail: filips.capligins@rtu.Iv \\ **e-mail: anna.litvinenko@rtu.lv
}

The paper presents a study of the chaotic jerk circuit (CJC) employment capabilities for digital communications. The concept of coherent chaos shift keying (CSK) communication system with controlled error feedback chaotic synchronization is proposed for a specific CJC in two modifications. The stability of chaotic synchronization between the two CJCs was evaluated in terms of voltage drop at the input of the slave circuit and the impact of channel noise using simulations and experimental studies.

Keywords: Chaotic communication, chaotic synchronization, chaos shift keying, experimental study, jerk circuit, secure communication system, Sprott circuit.

\section{INTRODUCTION}

Possible applications of chaotic signals in the field of digital communications have been studied since the early 90s. The main features of chaotic signals that are desirable for telecommunications include noise-like appearance, high entropy, spread spectrum, resistance to jamming and robustness in multipath environments [1].

A chaotic system (including a chaotic generator) is a deterministic nonlinear dynamic system that can be mathematically described by a system of differential equations (e.g., Lorenz system) or using discrete maps (e.g., logistic map). It can also be implemented using an electronic circuit like Chua's circuit.

This study focuses on a chaotic jerk circuit (CJC). Chaotic jerk systems and their circuit implementations were discovered only at the beginning of the $21^{\text {st }}$ century by 
Sprott [2], [3]. Jerk circuits are relatively simple and robust to parametric mismatch, so they are suitable candidates for practical implementation.

In general, a jerk is a third-time derivative of some variable $x$, which would correspond to the first-time acceleration derivative in a mechanical system. Thus, a jerk equation is a third-order time differential equation. Within the specific parameter range, jerk equations can provide chaotic solutions. For example, simple jerk equation:

$\dddot{x}+\ddot{x}+x+\alpha^{2} \exp \left(\frac{\dot{x}}{\alpha}\right)=0$

can provide chaotic solutions for $\alpha<0.27$, except for some small periodic windows [4]. The CJC can be considered an analogue computer that solves the jerk equation using electronic components with voltage or current as variables.

Another useful property of chaotic systems is chaotic synchronization, when one chaotic system (master) is tuned to another (slave) so that they produce the same outputs. Two separate identical chaotic systems will generate two completely uncorrelated signals if there are even smallest differences in initial conditions, but with chaotic synchronization, their dynamics can be tuned so that their trajectories in phase space become equal. If the master chaotic system is in the transmitter and slave - in the receiver, their synchronization can be used to provide secure communications that are usually extremely difficult to intercept or decode by any other party without having the exact receiver system [1].

Two chaotic circuits must be coupled in an appropriate way for synchronization to be possible. One of the first and simplest chaotic synchronization techniques proposed by Pecora and Carroll in 1991 [5] uses direct coupling of two identical chaotic systems. In this case, one of the slave system variables is replaced by the corresponding variable from the master system, forcing other slave system variables adjust their trajectories to master system variables. Another common technique is error feedback synchronization [6] - the instantaneous difference between the signal received from the master system and the signal from the slave system (which is composed using the same function as in the transmitter) produces an error signal which, through the feedback loop, modifies the state of the receiver to minimise the error. It has been shown [7] that linear error feedback synchronization provides better performance than Pecora-Carroll synchronization. In the present study, specific controlled error feedback synchronization [8] is implemented and discussed in Section 3.

Existing chaos-based communication systems can be divided into two classes: with coherent and non-coherent detection. In coherent detection schemes, synchronization is exploited to generate a copy of the transmitted chaotic signal at the receiver, which is then used in various techniques to reconstruct the transmitted data. In noncoherent detection schemes, synchronization is not used. Data recovery is performed by detecting some features of the received signal without regenerating any local chaotic signal on the receiver side or the need for channel state information [9]. Common examples of coherent modulation techniques are chaotic masking and chaos shift keying (CSK). For non-coherent modulation, examples include chaos on-off keying (COOK), differential chaos shift keying (DCSK) and correlation delay shift keying (CDSK).

Chaotic communication systems with non-coherent detection are easier to implement, but they mainly rely on additional data encryption algorithms for secure transmission. Coherent systems involve cha- 
otic synchronization for data transmission, which makes much higher levels of security possible, but achieving stable synchronization may be challenging.

In the present study, the CSK technique is chosen, and the concept of a communication scheme for CJC is proposed (see Section 4). For a typical CSK, the transmitter uses two different chaotic generators, one representing the " 1 " bit, the other - " 0 ". Active switching between both transmitting chaotic systems is performed for each corresponding bit of the digital data sequence. On the receiver side, two chaotic generators, identical to those on the transmitter, receive the transmitted signal. During the transmission of a single bit, the synchronization occurs only in one of the receiving chaotic generators. Estimation of the transmitted bit is performed by comparing the synchronization errors or correlation coefficients between both receiver generators. If the chaotic generators used to transmit both data bits produce chaotic signals with very similar statistical properties, such a communication system is hard to intercept and decode without having exact chaotic generators and knowing exact parameters used for transmission setup. The drawback of CSK is speed - the bit duration must be long enough for synchronization to take place.

Due to the novelty of CJCs, the number of presented studies is somewhat limited, especially in the field of possible applications of CJCs in communication systems. One study [10] proposes using CJC in the DCSK communication scheme, and another [11] - in the CDSK scheme. The possibilities of using two synchronized CJCs for a chaotic masking communication system are studied in [12]-[15]. The present study is the first to propose the concept of a coherent CSK communication system with controlled error feedback synchronization of the jerk circuits.

This paper is organised as follows. Section 2 briefly describes the two CJCs used - Sprott's original jerk circuit and its modification. In Section 3, the implemented controlled error feedback synchronization method is reviewed. The proposed CSK scheme is described in Section 4. In Section 5 , the synchronization stability between the same and different CJCs is investigated concerning voltage drop at the input of the slave circuit and the impact of channel noise. Finally, Section 6 provides conclusions.

\section{SPROTT CHAOTIC JERK CIRCUIT}

For the present study a specific CJC proposed by Sprott in [4] was chosen. This jerk circuit is a relatively simple chaotic oscillator that, unlike many other chaotic circuits, is robust to parameter variations and does not require careful tuning of component values. It uses a diode as a nonlinear element whose characteristics are not critical, and the only reactive components are three identical capacitors. As shown in Fig. 1, this CJC consists of three conse- quent operational amplifier integrators, an inverter, a diode, and two feedback loops. All resistors and all capacitors should be of the same nominal values, chosen as $R=10$ $\mathrm{k} \Omega$ and $C=100 \mathrm{nF}$ for this study. This CJC electronically implements a system of three first-order equations:

$$
\left\{\begin{array}{c}
\dot{x}=y \\
\dot{y}=z \\
\dot{z}=-z-x-10^{-9}\left[\exp \left(\frac{y}{0.026}\right)-1\right]
\end{array}\right.
$$


that can be decomposed from the general jerk equation (1) with slight modification, so that the exponential function describes a simplified mathematical model of a typical silicon diode.

Since the CSK modulation scheme requires two chaotic generators, a modification of this CJC (see Fig. 2) with an inverted diode direction is proposed. Mathematical expression (2) can be changed into:

$$
\left\{\begin{array}{c}
\dot{x}=y \\
\dot{y}=z \\
\dot{z}=-z-x+10^{-9}\left[\exp \left(\frac{-y}{0.026}\right)-1\right]
\end{array}\right.
$$

to represent the modified Sprott CJC. Further on, the first CJC will be addressed as a CJC with a direct diode, the second - as a CJC with an inverted diode.
For a visual comparison of both CJCs, a projection of the chaotic attractor was recorded from the prototype circuits (reviewed in Section 5) for the CJC with a direct diode (see Fig. 3) and for the CJC with an inverted diode (see Fig. 4). Both attractors exhibit similar dynamics, but for opposite voltage values.

Sprott CJC generates broadband chaotic signal. However, it has a dominant oscillation frequency, which can be approximately predicted [4] as follows:

$f_{\text {dom }}=\frac{0.792552}{2 \pi R C}$.

For the selected values of $R$ and $C$, this gives $126 \mathrm{~Hz}$, which is close to the dominant oscillation frequency observed in simulations and experiments.

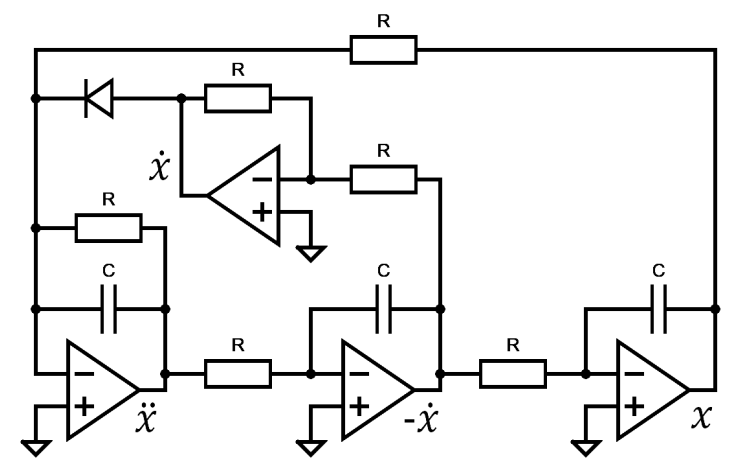

Fig. 1. Schematic of the Sprott CJC [4], with direct diode position.

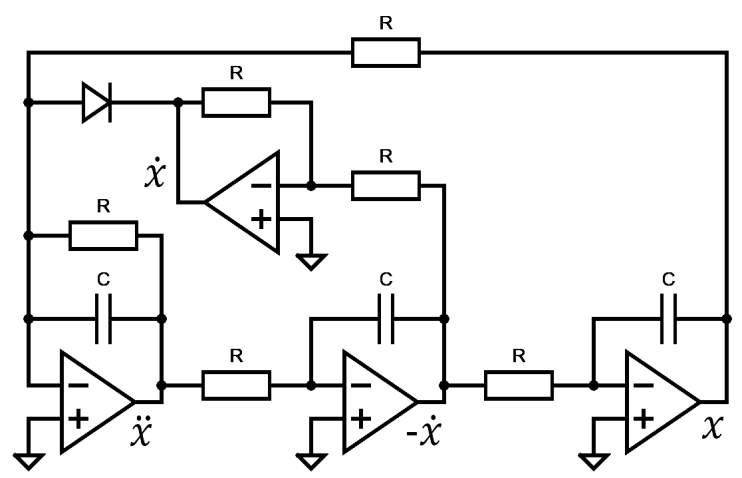

Fig. 2. Schematic of the modified Sprott CJC, with inverse diode position. 


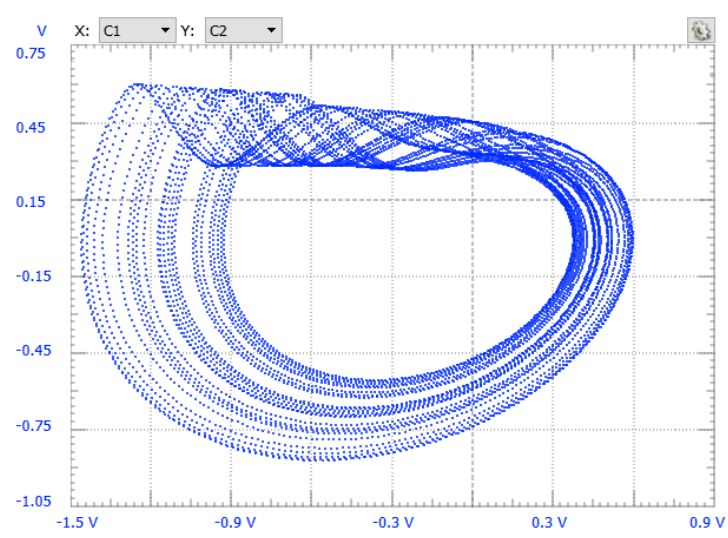

Fig. 3. Attractor projection in the phase plane, recorded from CJC prototype with direct diode position.

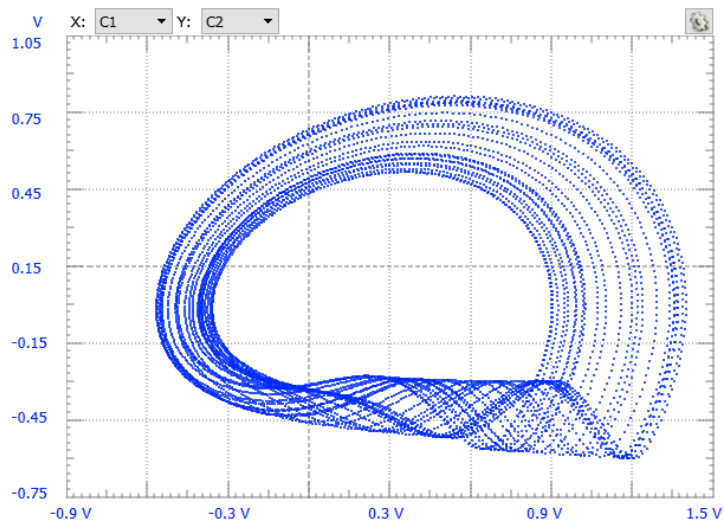

Fig. 4. Attractor projection in the phase plane, recorded from CJC prototype with inverse diode position.

\section{ERROR FEEDBACK SYNCHRONIZATION}

According to [8], two jerk circuits can be synchronized using a controlled error feedback method called practical finite-time synchronization. This method is designed explicitly for jerk circuits and aims at

$$
\left\{\begin{array}{l}
\dot{x}_{1}=x_{2} \\
\dot{x}_{2}=x_{3} \\
\dot{x}_{3}=a_{1} x+a_{2} x_{2}+a_{3} x_{3}+f\left(x_{1}, x_{2}, x_{3}\right)
\end{array},\right.
$$

whereas the slave CJC equation system:

$$
\left\{\begin{array}{l}
\dot{y}_{1}=y_{2}+k_{1} S \\
\dot{y}_{2}=y_{3}+k_{2} S \\
\dot{y}_{3}=a_{1} y+a_{2} y_{2}+a_{3} y_{3}+f\left(y_{1}, y_{2}, y_{3}\right)+k_{3} S
\end{array}\right.
$$


will have additional components $k_{i} S$ (synchronization controller) added for each state variable of the slave system. According to the equation:

$S=\sum_{i=1}^{3}\left(y_{i}-x_{i}\right)$,

$S$ is the sum of the differences between the state variables of both systems, which is also equal to the difference of the sums of the state variables of each system. If the $S$ value for coupled CJC reaches zero, both systems are synchronized, but in a practical synchronization, $S$ can only reduce to a small non-zero value. Further in this paper this difference is designated as a synchronization error, since its value directly corresponds to the difference between the states of the master and slave CJC. By employing the feedback, the synchronization error is applied to the slave system, but differently for each state variable. The coefficients $k_{i}$ are intended to individually modify the sign and/or strength of the synchronization error signal applied to each state variable of the slave system in a manner that ensures high convergence speed for the synchronization. Consequently, the dynamics of the slave CJC is modified in a way that reduces synchronization error when two identical jerk circuits are coupled.

This method was used for synchronization of the Sprott CJCs. As shown in Fig. 5, the sums of the state variables of each system and their differences (synchronization error) are calculated electronically using operational amplifiers. Essential parts of this synchronization scheme are resistors $R c 1, R c 2$ and $R c 3$, which implement the function of $k_{i}$ variables by controlling the amount of current from the synchronization error signal applied to each state variable (feedback strength).

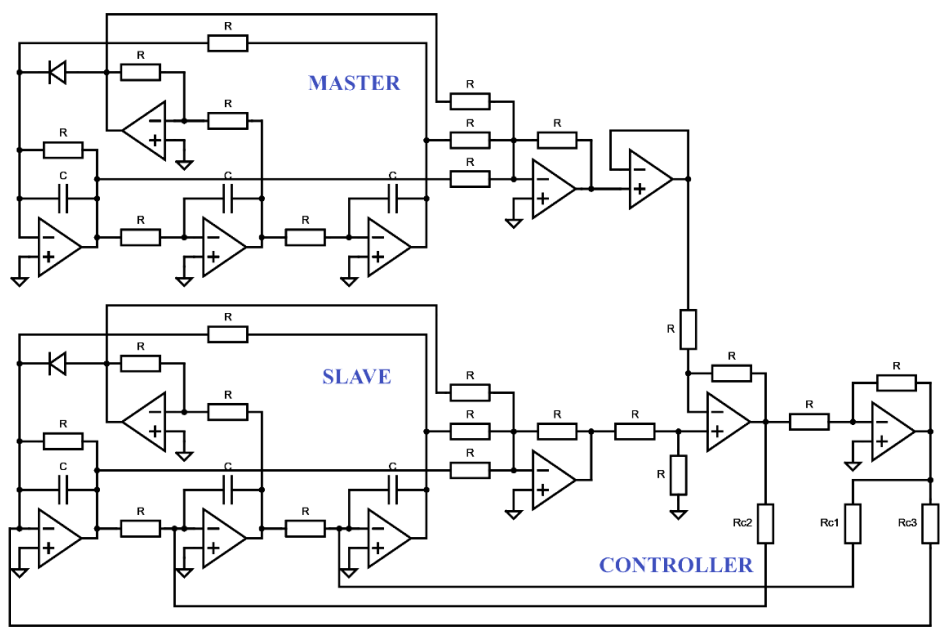

Fig. 5. Schematic of the synchronization circuit for two identical CJCs using the method presented in [8].

Resistance values that provide an acceptable quality of chaotic synchronization can be approximately calculated [8], but in this study, an empirical approach was chosen to determine appropriate resistance values. Using a prototype circuit with two synchronizable CJCs, the resistors were replaced with potentiometers, and the rms voltage of the synchronization error, as well as the correlation graph between the equivalent state variables on both (master and slave) CJCs, were observed with 
different resistance values. Satisfactory synchronization results were obtained for $R c 1=50 \mathrm{k} \Omega, R c 2=10 \mathrm{k} \Omega$ and $R c 3=500$ $\Omega$; therefore, these values were chosen for the further studies of synchronization stability, discussed in Section 5.

Synchronization simulation for two identical CJCs shows (see Fig. 6) that the time required for the synchronization error to converge to a small non-zero value is approximately $10 \mathrm{~ms}$ - the synchronization speed is related to the dominant frequency of the jerk circuit and, according to (4), the speed can be increased by decreasing $R$ or $C$ values to any preferred level. However, maintaining the stability of the electronic circuit becomes more challenging at higher frequencies.

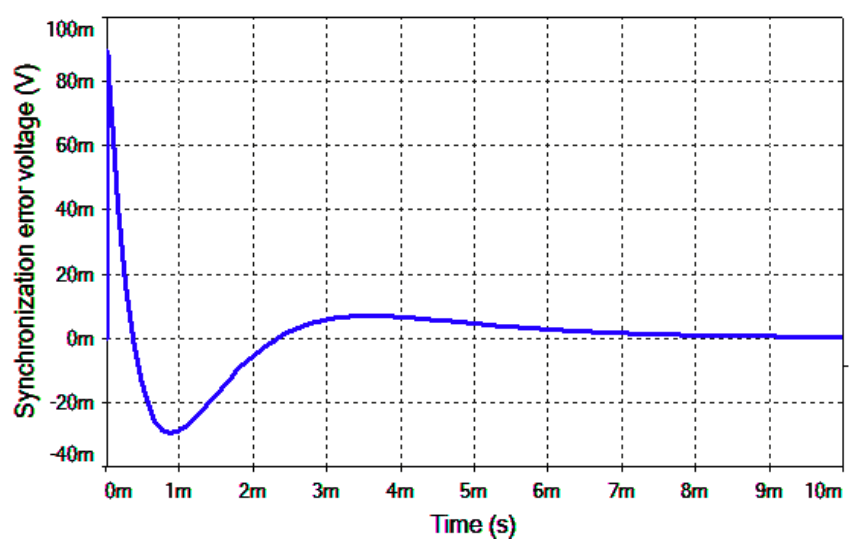

Fig. 6. Synchronization error signal reduction due to synchronization between master and slave CJCs with direct diode position and different initial conditions, obtained by Multisim simulation.

\section{CHAOS SHIFT KEYING}

It is notable that the error feedback synchronization method, described in the previous Section, works for two CJCs with direct diodes as well as for two CJCs with inverse diodes, without any additional tuning. Nevertheless, if two different CJCs are coupled in the proposed synchronization scheme, no synchronization occurs. To be more precise, some partial synchronization can be observed, but the rms voltage of the synchronization error is much larger than for two synchronized identical CJCs. This property plays a vital role in the proposed CSK communication system.

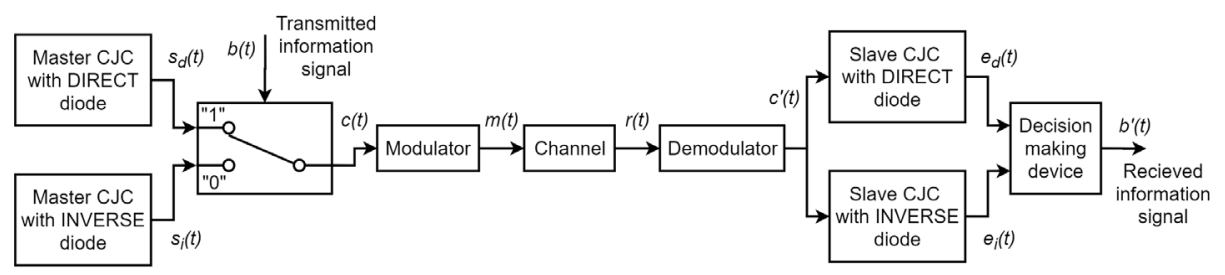

Fig. 7. Proposed coherent CSK communication system with controlled error feedback synchronization of jerk circuits. 
The proposed concept (see Fig. 7) is as follows. The transmitter has two CJCs, with a direct diode and with an inverted diode, which produces two different chaotic signals $s_{d}(t)$ and $s_{i}(t)$. Both chaotic signals are the sums of the three CJC state variables. The value of the transmitted binary information signal $b(t)$ is used to control a switch that selects the corresponding chaotic signal. The selected signal $c(t)$ should be modulated for transmission over the wireless channel. At the receiver the transmitted signal is demodulated and used in the synchronization scheme for both types of controlled slave CJCs, producing two synchronization errors $e_{d}(t)$ and $e_{i}(t)$ as outputs. The rms values of these errors, calculated over the bit duration period, must be compared to determine the value of the transmitted bit.

This communication system can be further simplified by reducing the number of CJCs required. For a transmitter, switching between two different jerk systems can be achieved by switching between two diodes with opposite directions in the same CJC. This approach can slightly reduce the size and complexity of the transmitter, but it may also reduce the maximal speed of the communication system given for the same bit error ratio due to the additional time spent on transients within the transmitting CJC. In a receiver, a single synchronizable slave CJC may be sufficient to estimate the transmitted bit using a threshold level of synchronization error, but such simplified detection can potentially reduce the noise immunity of the communication system.

To validate the proposed concept of the CSK communication system, the stability of synchronization error was evaluated under different conditions for all four possible master and slave CJC interconnections. The evaluation was made for a simplified system model, for a baseband equivalent, without additional modulation and wireless channel. The experimental setup and results are discussed in the next section.

\section{EVALUATION OF SYNCHRONIZATION STABILITY}

The synchronization properties of the studied CJCs were evaluated using software simulations and hardware prototype. The first part of this section describes the experimental setup and evaluates some basic electrical properties of the output signal for

\subsection{Setup}

Simulations were carried out using Multisim model (see Fig. 8) with a maximal time step of $10^{-4} \mathrm{~s}$. The structure of the model repeats the design of the prototype circuit (see Fig. 9). Both systems use TL074CD operational amplifiers, which are low cost, high speed, and maintain large gain bandwidth. A conventional 1N4001 both CJC types and synchronization errors at different CJC interactive connections. The impact of the slave input voltage drop on the synchronization error is assessed in the second part, and the impact of noise - in the last part of this section.

silicon diode is used, and a bipolar power supply of $\pm 5 \mathrm{~V}$ is applied to the operational amplifiers since the chaotic signal voltage does not exceed $\pm 2 \mathrm{~V}$. The prototype uses four (two per circuit part) low dropout voltage regulators to provide two stable $\pm 5 \mathrm{~V}$ power supplies from four $9 \mathrm{~V}$ batteries. 
Table 1. Properties of the Master CJC Output Signal

\begin{tabular}{|l|c|c|c|c|c|c|}
\hline \multirow{2}{*}{ Model } & \multicolumn{3}{|c|}{ CJC with direct diode } & \multicolumn{3}{c|}{ CJC with inverse diode } \\
\cline { 2 - 7 } & $\mathrm{rms}(\mathrm{ac}), \mathrm{V}$ & $\mathrm{dc}, \mathrm{mV}$ & $\mathrm{p}-\mathrm{p}, \mathrm{V}$ & $\mathrm{rms}(\mathrm{ac}), \mathrm{V}$ & $\mathrm{dc}, \mathrm{mV}$ & $\mathrm{p}-\mathrm{p}, \mathrm{V}$ \\
\hline Multisim & 0.45 & 4.2 & 1.1 & 0.45 & 4.2 & 1.1 \\
\hline Prototype & 0.56 & 276 & 2.05 & 0.53 & -266 & 1.95 \\
\hline
\end{tabular}

Table 2. Synchronization Errors for Different Circuit Connection Combinations

\begin{tabular}{|l|c|c|c|c|}
\hline \multirow{2}{*}{ Model } & \multicolumn{3}{|c|}{ Circuit connection type } \\
\cline { 2 - 5 } & $\begin{array}{c}\text { II sync. error rms, } \\
\mathrm{mV}\end{array}$ & $\begin{array}{c}\text { DD sync. error rms, } \\
\mathrm{mV}\end{array}$ & $\begin{array}{c}\text { ID sync. error rms, } \\
\mathrm{mV}\end{array}$ & $\begin{array}{c}\text { DI sync. error rms, } \\
\mathrm{mV}\end{array}$ \\
\hline Multisim & 0.05 & 0.05 & 210 & 210 \\
\hline Prototype & 0.79 & 0.60 & 530 & 37 \\
\hline
\end{tabular}

Some basic electrical properties of both types of master CJC output signals were evaluated (see Table 1). Simulation results are equal for both CJCs, but the prototype shows some differences. Simulations show a little dc value where as the prototype has a relatively significant dc value, which also has an opposite sign for different CJCs. This property of the prototype circuits is probably associated with a small parametric mismatch of the components used (and the imperfection of the operational amplifier) and should be considered an essential impact factor for understanding further experimental results.
Four combinations of interactive CJC connections - II, DD, ID, and DI - were studied, where the first letter denotes the type of master CJC (direct or inverse) and the second letter denotes the type of the slave circuit. According to Table 2, two CJCs of the same type produce much smaller synchronization error than two different coupled circuits, although the prototype has significantly different levels of synchronization error rms voltage for the ID and DI cases. Visual comparisons of synchronization errors for different cases of CJC connections are shown in Fig. 10.

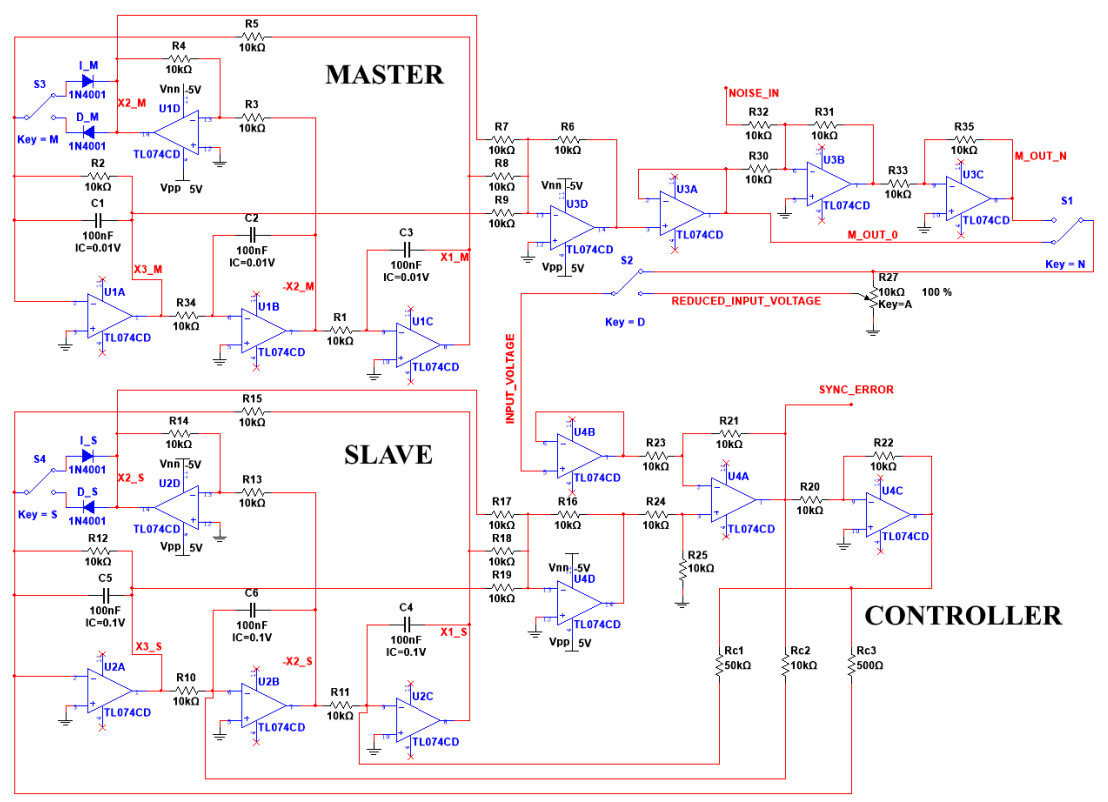

Fig. 8. Multisim model of two synchronizable CJCs with selectable noise addition and input voltage drop setup. 


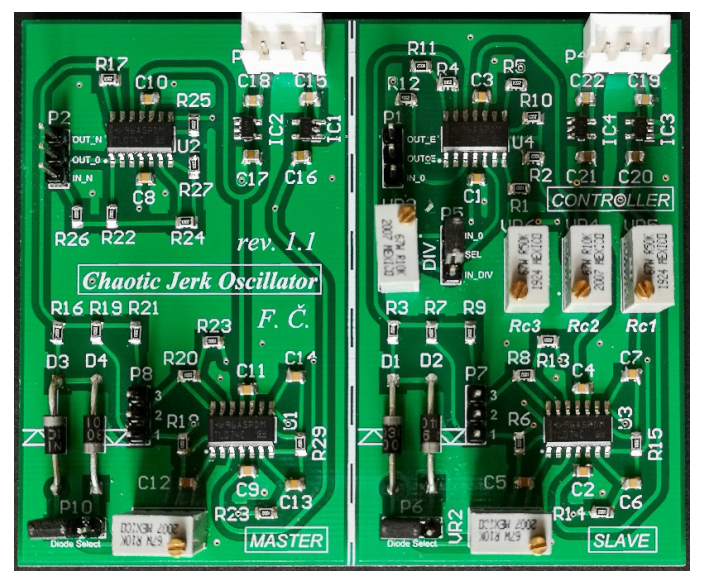

Fig. 9. Prototype circuit for evaluating the CJC synchronization properties, assembled on a printed circuit board.

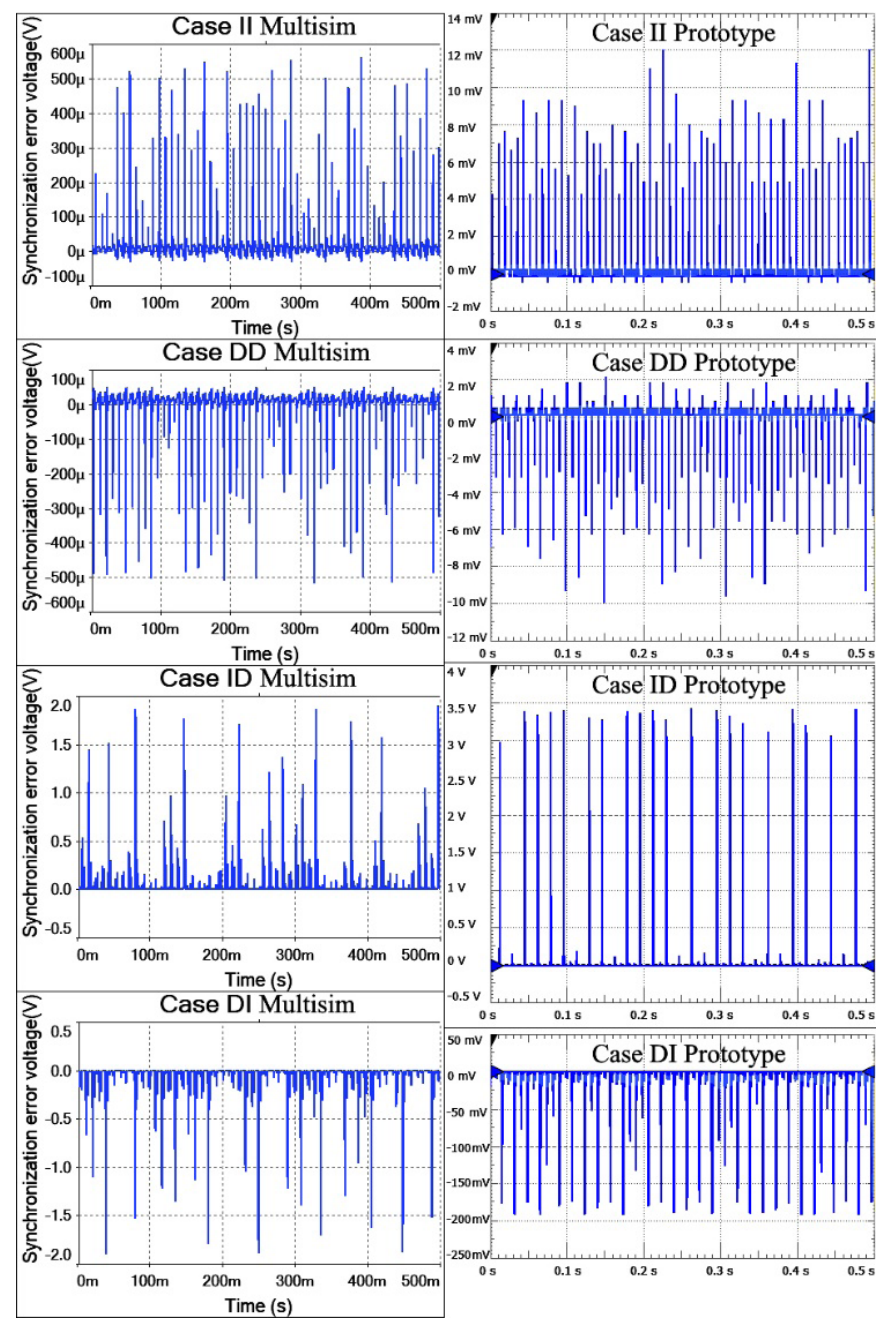

Fig. 10. Examples of simulated and experimentally measured synchronization error signals for all cases of possible CJC connections. 


\subsection{Impact of Input Voltage Drop}

In order to understand the importance of correct voltage level at the input of a controlled slave CJC, the sustainability of the synchronization error was evaluated as a function of the input voltage drop. A simple potentiometer-based voltage divider was used to linearly ramp down the output voltage of the master CJC before supplying it to the slave CJC controller (see R27 in Fig. 8).

Simulations of the slave CJC input voltage drop (see Fig. 11, a) show a distinct pattern of synchronization error dependence of input level between two equal and two different types of connected CJCs until the input signal drops to $-3 \mathrm{~dB}$ from the nominal level. Experimental measurements (see Fig. 11, b) also show significant differences between synchronization errors for the same and different CJCs in range until the input level of the voltage drops by -3 dB. Further decrease of the input voltage merges synchronization errors for all types of connections, making it impossible to use

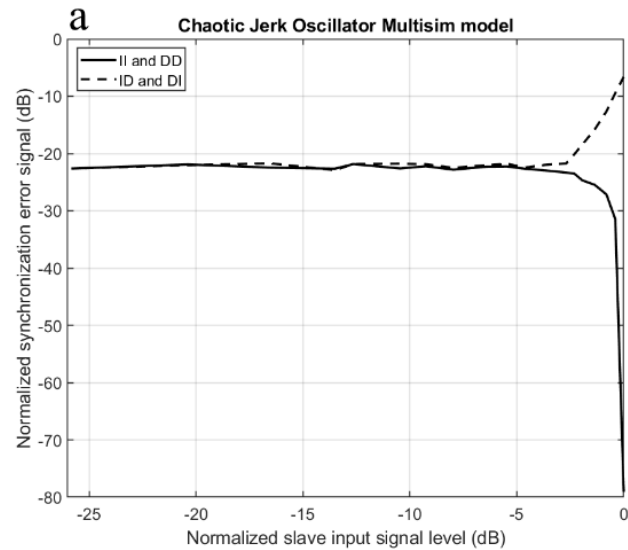

the CSK detection scheme.

A general pattern of the synchronization error signal drop together with the input signal is observed during both simulations and during experimental measurements, but this reduction is not caused by the improvement of synchronization quality. If zero voltage (or ground) is supplied to the controlled slave CJC, the synchronization error signal, as well as all state variables of the slave CJC, will decrease to a near-zero value such behaviour is predictable from the structure of synchronization scheme used (see Fig. 8). For this reason, the synchronization error rms voltage in Figs. 11 and 12 is normalized (divided) by the slave input rms voltage. In simulations, the normalized synchronization signal remains at the same level when the slave input signal drops below $-3 \mathrm{~dB}$, but in the prototype circuit, it is much less steady and increases slowly as the input voltage approaches zero.

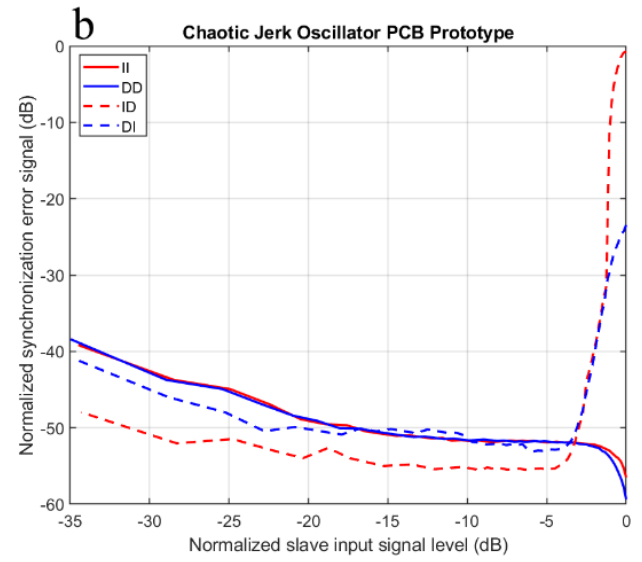

Fig. 11. Influence of slave input rms voltage level (normalized by its maximum value) drop on synchronization error rms voltage (normalized by the slave input rms voltage) for different combinations of CJC connections: $\mathrm{a}$ - simulated in the Multisim; b - measured in the prototype circuit.

If the slave input voltage level drops by $3 \mathrm{~dB}$, the synchronization error (for the cases II and DD) increases, which indicates a degradation of the synchronization qual- ity. In Multisim simulation, this degradation starts rather abruptly, but the prototype demonstrates much less synchronization loss at a slightly reduced input voltage. 
The significant difference between ID and DI types of CJC interconnections for the prototype becomes minimal after the input voltage drops below $-1.25 \mathrm{~dB}$. This fact helps understand that the relatively large synchronization error signal for the ID case is an instability issue in the slave CJC for this specific CJC connection. The slave CJC variables, along with the synchronization error signal, tend to jump to relatively

\subsection{Impact of Noise}

Noise resistance of the synchronization is evaluated by adding white noise to the output signal of the master CJC (see Fig. $8)$. The bandwidth of the transmitted chaotic signal is approximately $5 \mathrm{kHz}$, so the noise bandwidth is chosen the same. Simulation results (see Fig. 12, a) show very stable patterns for synchronized (II, DD) and desynchronized (ID, DI) cases of CJC connections that do not overlap or merge within the measured signal-to-noise ratios (SNR), whereas prototype measurements (see Fig. 12, b) show one case of overlap and less regular patterns.

In simulations, the synchronization error level for cases II and DD increases linearly with the noise level. However, in the experimental prototype, both cases show a different nonlinear pattern - for SNRs from 40 to $13 \mathrm{~dB}$, the synchronization error level raises considerably slower than in simulations. However, for SNR below $13 \mathrm{~dB}$, the case II error starts to increase almost linearly with noise, while in the DD case error increases much faster, almost reaching the error level of case ID at zero SNR. It is noteworthy that in the researched prototype, the slave CJC with a direct diode is much less stable. Similarly to the case ID in the previous experiment, when the input voltage in the DD case exceeds (due to the noise added) some specific level, the CJC high voltages (see Fig. 10) that are limited by the saturation voltage of the operational amplifiers. Decreasing the input voltage below $-1.25 \mathrm{~dB}$ no longer pushes the slave CJC to the divergent solutions. This issue is a peculiarity of the prototype circuit and may not repeat or may behave entirely differently in any other prototype circuit made according to the same design.

begins to produce divergent results that lead to short peaks of saturated voltage values. Experimental cases II and DI are more stable than ID and DD, and they resemble simulation results more closely. Different and significant dc voltage offsets, that the prototype CJCs have, possibly are one of the reasons for this instability issue observed in the experiments. It is not a desirable feature and should be avoided in a practical communication system.

Altogether, this experiment did not show any indications of a possible undistinguishable synchronization error for SNRs above $9 \mathrm{~dB}$ (when noise rms voltage is about three times less than chaotic signal rms voltage), which should enable the proposed CSK communication at these noise levels. Prototype improvements are required to verify the communication possibility at lower SNR levels, to ensure minimal (and equal) dc voltage bias for CJCs with direct and inverted diode. In addition, the actual minimal transmitted chaotic signal bandwidth that is necessary for a sustainable chaotic synchronization in an ideal environment was not estimated in this study, but such additional research would make it possible to improve the noise immunity of the system by using an appropriate bandpass filter at the input of the receiver. 

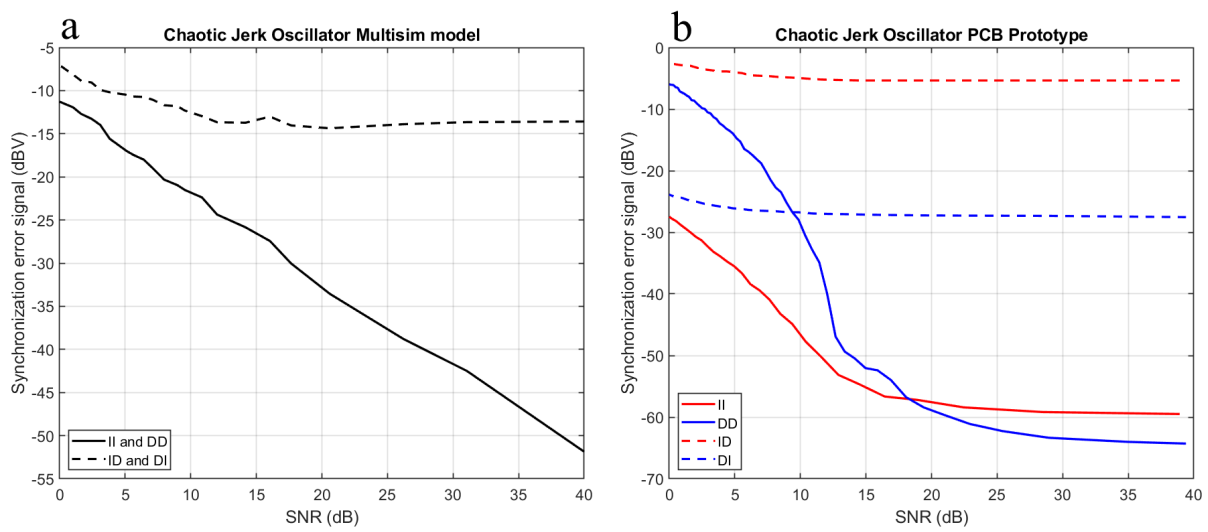

Fig. 12. Impact of signal-to-noise ratio on synchronization error rms voltage for different combinations of CJC connections: $\mathrm{a}$ - simulated in the Multisim; $\mathrm{b}$ - measured in the prototype circuit.

\section{CONCLUSIONS}

A coherent CSK communication system that uses two modifications of CJC with controlled error feedback synchronization has been proposed, and the stability of synchronization has been evaluated for different possible couplings between two equal and two different CJCs with respect to input voltage drop and channel noise, using simulations and a prototype circuit.

Synchronization error levels between synchronized (II, DD) and desynchronized (ID, DI) cases of CJC connections are distinguishable for the slave CJC input voltage level above $-3 \mathrm{~dB}$ from nominal value and for SNRs above $9 \mathrm{~dB}$.

The prototype circuit, created only for CJC synchronization evaluation, has identified several possible system weaknesses, such as de bias of the chaotic output signal and possible CJC instability issues, that should be carefully considered when designing and implementing the CSK communication system based on the synchronization of the jerk circuits.

According to the results, the proposed digital CSK communication system with CJC synchronization can be practically implemented. However, this study is just the first step in the engineering design process. Further steps of this research should involve a practical implementation of the proposed CSK communication scheme for the evaluation of the impact of noise, interference, multipath propagation, frequency shift and other issues that are common for wireless communication systems.

\section{ACKNOWLEDGMENTS}

The present research has been supported by Riga Technical University 2019/2020 project for strengthening scientific personnel capacity No. MP-2019/5, "Advanced Security Wireless Sensor Network Com- munication System with Chaotic Synchronization", and by the European Regional Development Fund within Activity 1.1.1.2 "Post-doctoral Research Aid" of the Specific Aid Objective 1.1.1 "To increase the 
research and innovative capacity of scientific institutions of Latvia and the ability to attract external financing, investing in human resources and infrastructure" of the Operational Program "Growth and Employment” (No. 1.1.1.2/VIAA/2/ 18/345).

\section{REFERENCES}

1. Stavroulakis, P. (2006). Chaos applications in telecommunications. CRC Press.

2. Sprott, J. C. (2000). A New Class of Chaotic Circuit. Phys. Lett. Sect. A Gen. At. Solid State Phys., 266 (1), 19-23. doi: 10.1016/ S0375-9601(00)00026-8.

3. Sprott, J. C., \& Linz, S. J. (2000). Algebraically Simple Chaotic Flows. Int. J. Chaos Theory Appl., 5 (2), 285.

4. Sprott, J. C. (2011). A New Chaotic Jerk Circuit. IEEE Trans. Circuits Syst. II Express Briefs, 58 (4), 240-243. doi: 10.1109/TCSII.2011.2124490.

5. Carroll, T. L., \& Pecora, L. M. (1991). Synchronizing Chaotic Circuits. IEEE Trans. Circuits Syst., 38 (4), 453-456.

6. Kolumbán, G., Kennedy, M. P., \& Chua, L. O. (1998). The Role of Synchronization in Digital Communications Using Chaos - Part II: Chaotic Modulation and Chaotic Synchronization. IEEE Trans. Circuits Syst. I Fundam. Theory Appl., 45 (11), 1129-114. doi: 10.1109/81.735435.

7. Pizolato, J. C., Romero, M. A., \& Neto, L. G. (2008). Chaotic Communication Based on the Particle-in-a-Box Electronic Circuit. IEEE Trans. Circuits Syst. I Regul. Pap., 55 (4), 1108-1115. doi: 10.1109/ TCSI.2008.916424.

8. Louodop, P., Kountchou, M., Fotsin, H., \& Bowong, S. (2014). Practical Finite-Time Synchronization of Jerk Systems: Theory and Experiment. Nonlinear Dyn., 78 (1), 597-607. doi: 10.1007/s11071-014-1463-5.

9. Kaddoum, G. (2016). Wireless Chaos-Based Communication Systems: A Comprehensive Survey. IEEE Access, 4, 2621-2648. doi: 10.1109/ACCESS.2016.2572730.

10. Liu, J., Sprott, J. C., Wang, S., \& Ma, Y. (2018). Simplest Chaotic System with a Hyperbolic Sine and its Applications in
DCSK Scheme. IET Commun., 12 (7), 809 815. doi: 10.1049/iet-com.2017.0455.

11. Rajagopal, K., Pham, V. T., Çiçek, S., Jafari, S., Karthikeyan, A., \& Arun, S. (2020). A Chaotic Jerk System with Different Types of Equilibria and its Application in Communication System. Teh. Vjesn., 27 (3), 681-686. doi: 10.17559/TV20180613102955.

12. Sambas, A., Ws, M. S., Mamat, M., Karadimas, N. V., \& Tacha, O. (2013). Numerical simulations in jerk circuit and its application in a secure communication system. In Recent Adv. Telecommun. Circuit Des. WSEAS 17th Int. Conf. Commun (pp. 190-196), 16-19 July 2013, Rhodes Island, Greece.

13. Sambas, A., Sanjaya, M., Mamat, M., \& Putra Prastio, R. (2016). Mathematical Modelling of Chaotic Jerk Circuit and its Application in Secure Communication System. Adv. Chaos Theory Intell. Control. Stud. Fuzziness Soft Comput. Springer, 337, 133-153. doi: 10.1007/978-3-319-30340-6.

14. Çiçek, S., Kocamaz, U. E., \& Uyaroğlu, Y. (2019). Secure Chaotic Communication with Jerk Chaotic System Using Sliding Mode Control Method and its Real Circuit Implementation. Iran. J. Sci. Technol. Trans. Electr. Eng., 43 (3), 687-698. doi: 10.1007/s40998-019-00184-9.

15. Nguemkoua Nguenjou, L. P., Kom, G. H., Mboupda Pone, J. R., Kengne, J., \& Tiedeu, A. B. (2019). A Window of multistability In Genesio-Tesi Chaotic System, Synchronization and Application for Securing Information. AEU - Int. J. Electron. Commun., 99, 201-214. doi: 10.1016/j.aeue.2018.11.033. 